\title{
PULL SYSTEMS WITH ADVANCE DEMAND INFORMATION
}

\author{
Ananth Krishnamurthy \\ David Claudio \\ Department of Decision Sciences and Engineering Systems \\ 110 8th Street \\ Rensselaer Polytechnic Institute \\ Troy, NY 12180, U.S.A.
}

\begin{abstract}
This paper investigates the benefits of integrating advance demand information (ADI) with pull-kanban type production and inventory control systems (PICS). Since efficient analytical models for performance evaluation studies of these systems are unavailable, we use simulation experiments for the study. In particular, we investigate the impact of several PICS design parameters such as kanban card limits, target finished goods inventory levels, amount of demand information available, and the quality of ADI on performance measures such as system throughput, inventory holding costs and customer service levels. Our study shows that in many situations, integrating ADI with pull systems provides opportunities for efficiencies that might be significantly greater that that available using pull systems alone. Further more, we show that the performance of systems operating under pull-type PICS with ADI could be fairly robust to the quality of information being shared.
\end{abstract}

\section{INTRODUCTION}

Recent advances in information technology such as ERP and EDI have enabled the sharing of information across supply chains. These advances have come at a time when supply chain partners are looking for new ways to collaborate and improve operational efficiencies to meet the ever increasing customer demand for customized products at reduced costs. Such collaborations are based on the underlying premise that increased information sharing will always result in greater efficiencies. While this belief is true, there is little detail on the exact nature of improvements that can be realized through such collaborations. Sharing information across supply chains require significant investments in information technology. Therefore, supply chain managers involved in information sharing efforts must have a clear understanding of the resulting benefits and the environments in which these benefits are obtained.
This research focuses on one important aspect of the benefit of information sharing - namely the sharing of advanced demand information (ADI) to improve supply chain efficiencies. In particular, it focuses on how ADI can be integrated with the production and inventory control systems (PICS) being adopted on the factory floor to improve operational efficiencies. Factory operations are complex and the efficiency of operations is sensitive to several factors such as machine utilization, inventory limits, and promised levels of customer service (Buzacott and Shanthikumar, 1993). Hence, it is hard to develop an intuition for PICS that would yield good performance. Over the last few decades, several PICS have been proposed to efficiently manage production resources, eliminate wasteful inventories, and improve customer service levels. Among these the most popular ones are the pull-type PICS such as base stock system, kanban system, and CONWIP. These systems have been successfully implemented in several industries and have therefore been the focus of several research studies too. However, none of the above mentioned pull-type PICS fully exploit the potential benefits of ADI shared across supply chains. A recent study by Karaesman et al. (2002) has shown that integration of advance demand information (ADI) with base-stock-type control policies could lead to significant improvement in efficiencies. Our research compliments this study by investigating the performance of PICS that integrating ADI with kanban type pull systems. In particular, we investigate the impact of design parameters such as target WIP levels or kanban card limits, target finished goods inventory levels, demand information lead times, and the quality of advance demand information on different performance measures. Since efficient analytical models that permit such a study are not yet available, we conduct simulation experiments to obtain necessary insights. Our study provides several insights with respect to the impact of integrating ADI with pull type PICS. We observe that in many situations, such integration provides opportunities for efficiencies that are greater than that possible using pull systems without ADI. 


\section{Krishnamurthy and Claudio}

Further more, we observe that there are several situations, where the performance of such systems could be fairly robust to variations in the quality of ADI being shared. Such insights could be used to justify collaboration efforts that will lead to increased overall supply chain efficiencies.

The rest of the paper is organized as follows. Section 2 provides a brief review of the literature. Section 3 describes the system being studied. Section 4 provides a description of the simulation set up and design of experiments. Section 5 provides a summary of the results obtained for systems operating under pull type PICS with perfect ADI. Insights with respect to imperfect in ADI are described in Section 6. Section 7 provides a summary of the results and discusses extensions of the current research.

\section{LITERATURE REVIEW}

Production and inventory control systems such as the base stock policy, MRP systems, and kanban control systems (KCS) have been the subject of intensive research for several years. Buzacott and Shanthikumar (1993), Hopp and Spearman (1996) and Zipkin (2000) provide an excellent summary of design and performance issues related to these systems. Among these systems the kanban control systems is perhaps the most popular. One of the key reasons is that the system is simple to implement and yet very efficient. Kanban control systems explicitly limit inventories using kanbans (cards) - when one unit is consumed from inventory, a kanban signal is sent upstream to begin work to replenish this inventory. Prior research such as that reported in Hopp and Spearman (1996) have shown that limiting inventories and triggering production in this manner yields better system performance when compared to traditional $\mathrm{MRP} /$ push systems. Alternative pull systems such as the CONWIP system (Hopp and Spearman, 1996), Generalized Kanban Control System (GKCS) and the Extended Kanban Control System (EKCS) discussed in Buzacott and Shanthikumar (1993), Zipkin (2000), and Liberopoulos and Tsikis (2003) also have similar advantages.

However, it must be noted that all pull systems (KCS, CONWIP, GKCS, EKCS) trigger production to replenish inventory already consumed. All customer demands are expected to be satisfied from stock, and information about future demands may or may not be explicitly used to determine appropriate stocking levels. Recent studies have argued that with increased supply chain collaboration and information sharing, greater efficiencies can be obtained via hybrid PICS that integrate advanced demand information (ADI) with pull control (Buzacott and Shanthikumar, 1993; Karaesman, et al., 2002; Krishnamurthy et al., 2004). Some of these studies (Krishnamurthy et al., 2004) also suggest that in manufacturing environments with high product variety pull systems could be inefficient and hybrid PICS might be imperative. In addition to imposing upper bounds on the WIP and finished goods inventory in the system hybrid PICS such as PAC (Buzacott and Shanthikumar, 1993), hybrid CONWIP, (Hopp and Spearman, 1996) include mechanisms to incorporate information about future demands and planned lead times to determine the release of production orders into different manufacturing stages. While there are several qualitative arguments to support the claim that integration of ADI with pull could help improve factory efficiencies, little is known about how such integration affects performance measures such as average level of work in process (WIP) material, average number of backorders and average level of finished goods inventories. It is also not clear to what extent the benefits depend on the quality of information being shared across supply chains. Our research provides some initial insights in this area.

Our work differs from prior studies on the impact of information sharing such as those reported in Hariharan and Zipkin (1995) and Gallego and Ozer (2001) in two respects. First, we explicitly consider the effect of finite production capacity and queuing effects in quantifying the performance PICS that incorporate ADI. Second, we investigate how the benefits from ADI are sensitive to variations in the quality of ADI used. A recent study that is most relevant to this work is a study by Karaesman et al. (2002) that explores the value of ADI for the single stage continuous time make to stock queue. Karaesman et al. (2002) show that ADI helps to reduce stock levels only when system loads are reasonable (not too high, not too low). However, their study assumes perfect information. By considering imperfect information and pull/kanban type PICS, this research compliments the findings presented in Karaesman et al. (2002).

Analytical models of systems with imperfect information can be quite complex due to the underlying stochasticity. As a first step towards understanding such systems, and in order to justify investing in efforts to develop such models, we conduct simulation studies to assess the impact of quality of ADI on performance of PICS. Our study yields several interesting performance insights on PICS and the quality of ADI and also suggests several opportunities for future research.

\section{SYSTEM DESCRIPTION}

In this section we describe the manufacturing system being studied and list the assumptions made in our analysis. In particular, we describe system operation under three different PICS (i) pull PICS without ADI, (ii), pull PICS with perfect ADI, and (iii) pull PICS with imperfect ADI.

\subsection{System Description}

We consider a system with three manufacturing stages in series (denoted by $\mathrm{M}_{\mathrm{i}} \mathrm{i}=1,2,3$ ). See Figure 1 . Products are sequentially processed in these three manufacturing stages. 


\section{Krishnamurthy and Claudio}

It is assumed that the manufacturing stages have finite production capacities, implying that products could potentially queue in the buffers between the stages and wait for the resources in a manufacturing stage. The output buffer at the end of stage 3, denotes the finished goods inventory buffer. We assume that there is sufficient supply of raw material at the beginning of stage 1. External customer orders arrive one at a time and are satisfied whenever possible from units available in the finished goods inventory buffer. If units are not available, customer requests are backordered and are satisfied immediately upon availability of inventory. Next we describe the operation of the system under different PICS.

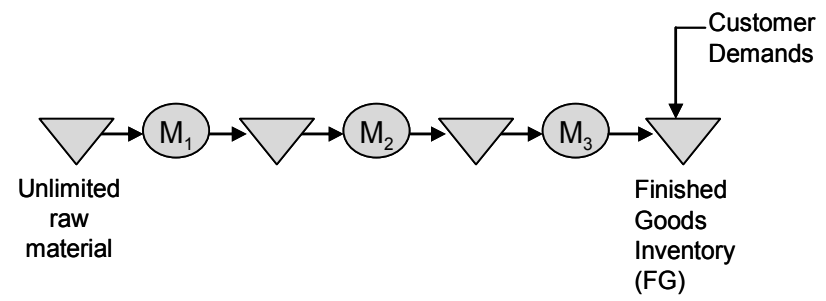

Figure 1: Manufacturing System

Pull systems without ADI: When the system operates under a pull based PICS without ADI, we assume that the inventory in the system is limited by the total number of kanban cards, denoted by K. Note that this inventory limit is for the total inventory in the system and not at each manufacturing stage. In other words, we assume that the pull control is of the CONWIP type (Hopp and Spearman, 1996). In the CONWIP form of pull control the kanban cards could either be attached to finished parts in the finished goods buffer or be attached to semi-finished parts in the different stages and buffers of the manufacturing system. The system then operates as follows. Customer orders are satisfied from finished goods, i.e., when an external order arrives, and if there is at least one item in the finished goods (FG) buffer, then it is used to satisfy the order. At the same time, the kanban card attached to the item is sent back to the beginning of the manufacturing stage 1 authorizing production. The kanban card together with the raw material queue for its turn at stage 1 and sequentially get processed in stages 1,2 and 3 before waiting in the finished goods buffer. If upon order arrival, there are no items in the FG buffer, it is backordered and the order waits until an item is available. See Figure 2. The dynamics of the system implies that in the pull system the total work in process inventory is at most equal to the total number of kanbans, K. Similarly, the system also ensures that the number of units in the finished goods inventory is at most equal to $\mathrm{K}$. The inventory limit, $\mathrm{K}$ and the characteristics of the demand and manufacturing processes determine the performance measures such as the system throughput, manufacturing lead times and the number of backordered demands. For simplicity, we assume that order arrival process is Poisson with rate $\lambda$ and that manufacturing station $\mathrm{M}_{\mathrm{i}}$ has exponentially distributed service times with mean $\mu_{\mathrm{i}}^{-1}$.

Pull systems with ADI: Next we consider the system operating under pull based PICS with perfect ADI. We assume that each customer places an order with the manufacturing facility $\tau$ time units in advance of their actual requirement (due date). We call $\tau$ the demand information lead time. For the case of perfect ADI, we assume that this lead time is constant and same for all customer orders. Further, we assume that the orders once placed by customers are not cancelled or changed. Note that the demand lead time $\tau$, is external to the manufacturing system, i.e. decided by the customer and is different from the planning lead time, LT that corresponds to the lead time used by the system to plan production. The planning lead time typically depends on the average manufacturing flow time - the average time taken to actually manufacture a unit.

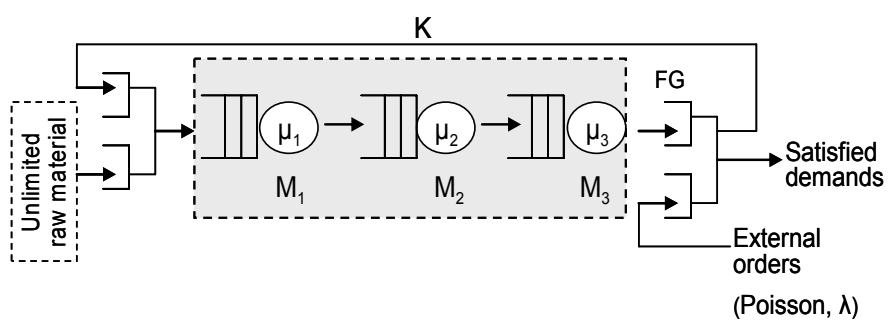

Figure 2: Manufacturing System Operating under Pull PICS

In view of the available advance demand information the manufacturing system might be able to meet customer demand with less finished goods inventory than that required in a system operating under pull PICS without ADI. For instance, if the demand lead time $\tau$ is significantly greater than the planning lead time, the system might not need to have any finished goods inventory. In other words, the advance demand information can be used to make the system operate in a make-to-order mode, i.e. raw material release is timed such that a finished product is available just in time to meet customer requirements at the due date.

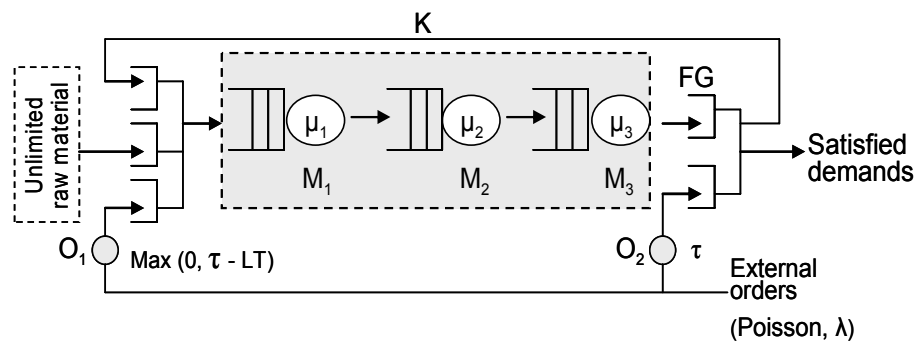

Figure 3: System Operating under Pull PICS with ADI 
To model these effects precisely in our simulation we augment our model by introducing order delay stations $\mathrm{O}_{\mathrm{i}}$, $\mathrm{i}=1$, 2. See Figure 3. At order delay station $\mathrm{O}_{1}$, the manufacturing system introduces a delay $T_{1}=\max (0, \tau-\mathrm{LT})$, between when an order is received and when it is activated upstream to trigger production via raw material release. Raw material is released for production, when an activated order and free kanban are available upstream of stage 1. As with the pull system without ADI, the kanban card stays with the part throughout the manufacturing process. At order delay station $\mathrm{O}_{2}$, the customer order undergoes a delay, corresponding to the demand lead time before manifesting as a customer demand on the due date, i.e. $\mathrm{T}_{2}=\tau$. On the due date, if there is a unit in the finished good inventory the demand is satisfied and the corresponding kanban card is released. If there are no units in the finished goods, the demand is backordered. Note that when $0<\tau<\mathrm{LT}$, it is likely that on the due date, a particular customer demand is satisfied from existing finished goods inventory and the production triggered by this order is merely used to replenish the inventory consumed to fulfill that order. In other words, depending upon whether the actual manufacturing flow time is greater or less than $\tau$, the customer orders might be satisfied from stock or by making products to order. Clearly, a system with $\tau=0$ corresponds to the case with no ADI and the manufacturing system must operate in a make-to-stock mode. The factors affecting system performance are the demand lead time, $\tau$, and planning lead time, LT, the number of kanbans, K, and the characteristics of the manufacturing processes and demand arrivals. Note that the system described above is closely related to the policy C described in Liberopoulous and Tsikis (2003) and that under certain limiting conditions of the parameters $(\tau$, LT, K), the hybrid PICS described above reduces to the other PICS, such as pull-kanban $(\tau=0, \mathrm{~K}<\infty)$, and MRP $(0<\tau<\infty, \mathrm{K}=\infty)$ systems.

Pull systems with imperfect ADI: Finally, we consider the system operating under pull type PICS with imperfect ADI. Imperfections in ADI could result due to randomness in demand lead times, discrepancies between order quantities and actual demand, discrepancies in quoted due dates and actual need dates. In this paper, we report findings related to one form of imperfection in ADI, namely, randomness in demand information lead times. The operation of the system is similar to that with perfect ADI, except for one key difference - in the case of imperfect ADI, the demand information lead times for all customers are not constant and equal to $\tau$. Although customers try to place orders $\tau$ time units before the due date, the exact timing of their order placement is random. In other words, demand information lead times of individual customer orders are sampled from a probabilistic distribution with mean $\tau$. For the purpose of this study, we assume that this distribution is exponential, since this choice allows us to study the impact of the high degree of variability that might exist in demand information lead times.

\section{SETUP OF SIMULATION EXPERIMENTS}

In this section, we describe the details of the simulation experiments. Section 4.1 describes the details of the simulation model when the system operates under pull strategy with ADI. Section 4.2 lists the experimental factors and describes the design of the simulation experiment.

\subsection{Simulation of Pull Strategy with ADI}

The simulation experiment conducted as part of this study was done using Arena ${ }^{\circledR}$ simulation software, version 5.00.02. (For more information on Arena ${ }^{\circledR}$ visit www.arenasimulation.com.) We describe the simulation model in detail for the system operating under a pull system with perfect ADI and comment on how this model is different from the simulation models for the other two PICS. The simulation model consists of three Create blocks, one for the orders, one for the kanban cards at the FG area, and the other for the kanban cards at the beginning of the stage 1. The three Create blocks generate the entities needed at time zero. The orders created are immediately sent to a Process block where the orders are released into the system after exponentially distributed time intervals with mean $\lambda^{-1}$. When an order is released, a Separate block simultaneously creates a duplicate of the order. The first copy is sent to the delay node $\mathrm{O}_{2}$, and the other copy is sent to the node $\mathrm{O}_{1}$ where it becomes an activated order after waiting for a duration corresponding to $\max (0$, $\tau$ - LT) time units. Both nodes $\left(\mathrm{O}_{1}\right.$ and $\left.\mathrm{O}_{2}\right)$ are represented by Process blocks. Once released from the node $\mathrm{O}_{1}$ at the beginning of the station, the activated order is routed to a Match block that models a fork/join station. At this block, an activated order, a kanban card, and a raw material unit, are matched before being routed to the queue at station $\mathrm{M}_{1}$.

The processes at stations $\mathrm{M}_{1}, \mathrm{M}_{2}$, and $\mathrm{M}_{3}$, are simulated using three Process blocks. Each process block is comprised of an infinite queue and an exponential server with mean service time equal to 1 . Each entity waits in queue at machine $\mathrm{M}_{\mathrm{i}}$, while the server empties the queue using a FCFS discipline. Once at the machine, an entity seizes the machine and undergoes an exponential delay prior to releasing the resource. Subsequent to departure from Machine 3, an entity enters a Match block where a unit in FG is matched with a customer demand. When the match occurs, at a Separate block the demand and the kanban are separated. The demand leaves the system with a unit of inventory, and the kanban card is sent back to the beginning of the station $\mathrm{M}_{1}$. A series of Assign and Record blocks are used to record the parameters of interest.

The simulation model for a system operating under pull system without ADI is similar, except that in this case, 


\section{Krishnamurthy and Claudio}

we set $\tau=0$. For a system operating under pull system with imperfect ADI, corresponding to each customer order we define an attribute corresponding to its demand information lead time. The value of this attribute is drawn from an exponential distribution with mean $\tau$ and this value is used to determine the delays at $\mathrm{O}_{1}$ and $\mathrm{O}_{2}$ corresponding to this order. Figure 4 provides a display of the system model in Arena. Note that in the figure, we omit displaying some of the record blocks for simplicity.

\subsection{Design of the Simulation Experiments}

The simulation experiments focus on the impact of four key factors and their effect on different measures of system performance. These include: (i) number of kanban cards, $\mathrm{K}$, (ii) the arrival rate of orders, $\lambda$, (iii) the target level finished goods inventory, FG, and (iv) demand information lead time, $\tau$. For the number of kanban cards we use three levels, $\mathrm{K}=5,10,20$. For the arrival rate of orders we use four levels of $\lambda=0.5,0.6,0.7$, and 0.8 . For the initial level of finished goods inventory, we consider three levels, 0 , $\mathrm{K} / 2$ and $\mathrm{K}$. Note that this implies that the initial inventory at $\mathrm{FG}$ for these three cases will be equal to $0, \mathrm{~K} / 2$ and $\mathrm{K}$ respectively. When the initial $F G$ inventory is $x(x=0, K / 2$, $\mathrm{K}$ ), we assume that all the remaining $\mathrm{K}-\mathrm{x}$ kanbans are in stage 1 . This yields 9 cases in total. When $\mathrm{K}=5$, initial $\mathrm{FG}$ inventory could take values 5,3 , and 0 respectively. Similarly when $\mathrm{K}=10$, initial $\mathrm{FG}$ inventory could take values of 10,5 , and 0 respectively, and finally when $\mathrm{K}=20$, initial FG inventory could take values of 20,10 , and 0 respectively. For pull systems with ADI, we also vary the amount of demand information lead time, $\tau$. We use seven levels corresponding to $\tau=0$ (no ADI), $0.25 \mathrm{LT}, 0.5 \mathrm{LT}, 0.75 \mathrm{LT}$, $1 \mathrm{LT}, 1.5 \mathrm{LT}$, and $2 \mathrm{LT}$. When perfect ADI is available and $\tau=2 \mathrm{LT}$, then information regarding each demand is obtained exactly 2LT time units in advance. If demand information is not perfect, then for the same situation, demand information is obtained $\tau$ time units in advance, where $\tau$ is drawn from an exponential distribution with mean 2LT.

In all our experiments, we assume that the service times at the machines have an exponential distribution with mean, $\mu_{\mathrm{i}}^{-1}=1, \mathrm{i}=1,2,3$. We also assume that arrival process of demand and orders are Poisson. These assumptions are not critical in a simulation model, but have been made to enable comparison with future analytical queuing models. In summary, we have a general full factorial experimental design with four factors. Table 1 represents all the experiments for the case of pull systems with perfect ADI and $K=5$. Similar sets of experiments are used for $K=10$ and $\mathrm{K}=20$. These result in an overall set of 252 experiments for the case of systems operating under pull PICS with perfect advance demand information. In Table 1, a kanban allocation labeled "K-i" denotes the case where the initial finished goods inventory level is i. For each experi- ment we run 50,500 units through the system. In each run, the statistics corresponding to the first 500 orders are discarded to account for transient start-up effects.

The estimates of the lead time, LT for the experiments with ADI are obtained simulation results for the corresponding experiment with no ADI. For example, the first set in Table 1 corresponds to $\mathrm{K}=5, \lambda=0.8$, and an initial finished goods level of 5 . We run this simulation experiment with $\tau=0 \mathrm{LT}$ and record the average manufacturing lead time for that case over all the replications. We set LT to this average value and use this value to compute the corresponding order delays at $\mathrm{O}_{1}$ for the cases where $\tau=0.25$ LT, 0.25 LT, 0.5 LT, 0.75 LT, 1 LT, 1.5 LT, and 2 LT. For systems with imperfect ADI, we run simulations for a restricted set of experiments. In particular, we run simulations for $\lambda=0.5$ and 0.8 , with $\tau$ varying from $0.25 \mathrm{LT}$ to 1 LT. The finished goods allocation considered are similar to that considered for the case with perfect ADI. The metrics of interest are the total WIP, average number of units in the FG area, and average number of backorders.

\section{PULL SYSTEMS WITH PERFECT ADI}

This section discusses the simulation results related to the systems with perfect ADI. We discuss the impact of key parameters on the performance of pull systems with perfect ADI. Subsequently, in Section 6 we analyze the impact of imperfect ADI on system performance.

\subsection{Results and Insights}

Our first set of results demonstrates the impact of ADI on the average total work in process (Total WIP) inventory in the system. We define the average Total WIP as the average number of units in Stages 1, 2 and 3, i.e. we do not include the average number of units in the finished goods inventory. Figure 5 plots the variation of Total WIP with system load, denoted by $\lambda$ for three different values of $K$, namely, $K=5,10$ and 20 . For each value of $K$, we consider the three levels of initial finished goods inventory, $\mathrm{K}, \mathrm{K} / 2$ and 0 respectively, and 7 cases of ADI with $\tau$ ranging from is $0 \mathrm{LT}$ to $2 \mathrm{LT}$. This implies that for each value of K, Figure 5 plots the variation of total WIP with $\lambda$ for 21 possible scenarios. From the figure we notice that as expected, the total average WIP increases with increase in K. Additional kanbans allow the system to achieve higher throughput, but this higher throughput also implies higher WIP inventories. However, the figure shows that the total average WIP is fairly insensitive to initial finished good allocation or amount of ADI. This is to be expected since WIP inventories are primarily a function of the system load.

On the other hand, Figures 6 (a) and (b) demonstrate an important effect of integrating ADI into pull systems. Figure 6(a) plots the variation of average number of backorders, denoted by $\mathrm{BO}$ with system load denoted by $\lambda$. 


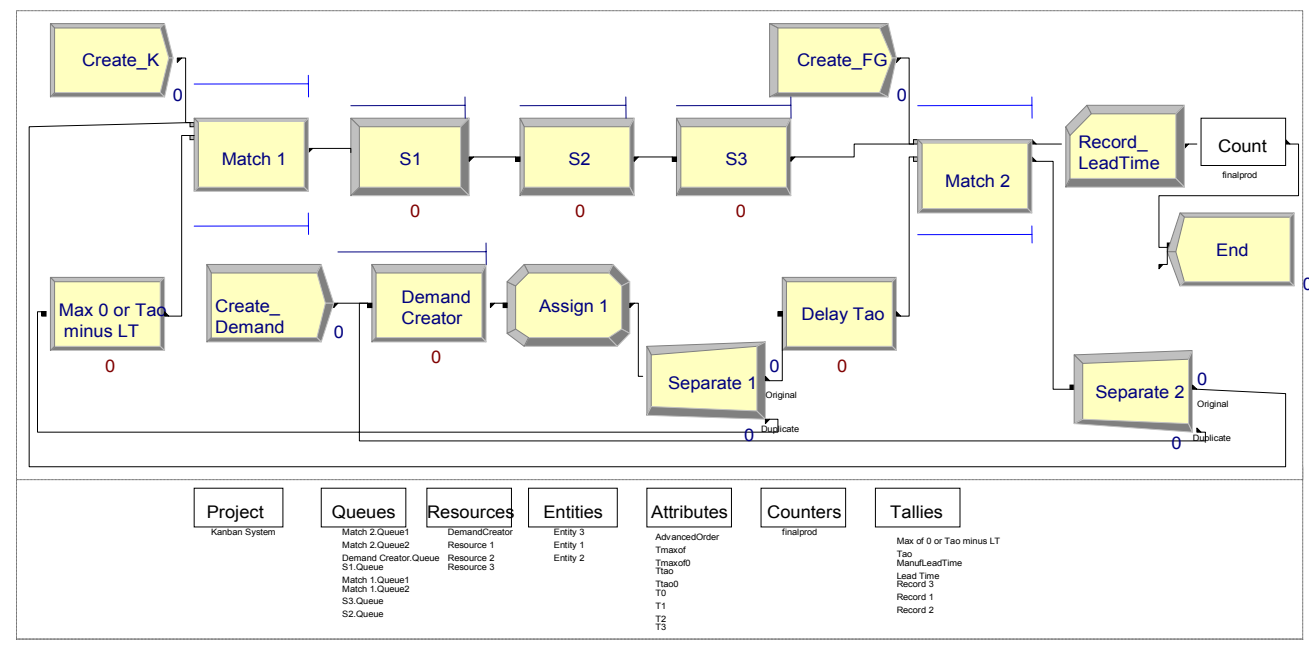

Figure 4: Simulation Model in Arena

Table 1: List of experiments when $\mathrm{K}=5$

\begin{tabular}{|c|c|c|c|c|c|c|c|c|c|c|c|c|}
\hline \multirow[b]{2}{*}{ K } & \multicolumn{3}{|c|}{ Allocation } & \multicolumn{3}{|c|}{ Allocation } & \multicolumn{3}{|c|}{ Allocation } & \multicolumn{3}{|c|}{ Allocation } \\
\hline & $\lambda$ & of $K$ & $T$ & $\lambda$ & of $K$ & $T$ & $\lambda$ & of $K$ & $T$ & $\lambda$ & of $K$ & $T$ \\
\hline 5 & 0.8 & $0-5$ & OLT & 0.7 & $0-5$ & $\begin{array}{ll}0 \mathrm{LT} \\
\end{array}$ & 0.6 & $0-5$ & OLT & 0.5 & $0-5$ & $0 \mathrm{LT}$ \\
\hline 5 & 0.8 & $0-5$ & $0.25 \mathrm{LT}$ & 0.7 & $0-5$ & $0.25 \mathrm{LT}$ & 0.6 & $0-5$ & $0.25 \mathrm{LT}$ & 0.5 & $0-5$ & $0.25 \mathrm{LT}$ \\
\hline 5 & 0.8 & $0-5$ & $0.5 \mathrm{LT}$ & 0.7 & $0-5$ & $0.5 \mathrm{LT}$ & 0.6 & $0-5$ & $0.5 \mathrm{LT}$ & 0.5 & $0-5$ & $0.5 \mathrm{LT}$ \\
\hline 5 & 0.8 & $0-5$ & $0.75 \mathrm{LT}$ & 0.7 & $0-5$ & $0.75 \mathrm{LT}$ & 0.6 & $0-5$ & $0.75 \mathrm{LT}$ & 0.5 & $0-5$ & $0.75 \mathrm{LT}$ \\
\hline 5 & 0.8 & $0-5$ & $1 \mathrm{LT}$ & 0.7 & $0-5$ & \begin{tabular}{l|l|}
$1 \mathrm{LT}$ \\
\end{tabular} & 0.6 & $0-5$ & $1 \mathrm{LT}$ & 0.5 & $0-5$ & 1 $1 \mathrm{LT}$ \\
\hline 5 & 0.8 & $0-5$ & $1.5 \mathrm{LT}$ & \begin{tabular}{|l|l|}
0.7 \\
\end{tabular} & $0-5$ & $1.5 \mathrm{LT}$ & \begin{tabular}{|l|}
0.6 \\
\end{tabular} & $0-5$ & $1.5 \mathrm{LT}$ & 0.5 & $0-5$ & $1.5 \mathrm{LT}$ \\
\hline 5 & 0.8 & $0-5$ & $2.0 \mathrm{LT}$ & 0.7 & $0-5$ & $2.0 \mathrm{LT}$ & 0.6 & $0-5$ & $2.0 \mathrm{LT}$ & 0.5 & $0-5$ & $2.0 \mathrm{LT}$ \\
\hline 5 & 0.8 & $2-3$ & $0 \mathrm{LT}$ & 0.7 & $2-3$ & OLT & 0.6 & $2-3$ & $0 \mathrm{LT}$ & 0.5 & $2-3$ & $0 \mathrm{LT}$ \\
\hline 5 & 0.8 & $2-3$ & $0.25 \mathrm{LT}$ & \begin{tabular}{|l|l|}
0.7 \\
\end{tabular} & $2-3$ & $0.25 \mathrm{LT}$ & 0.6 & $2-3$ & $0.25 \mathrm{LT}$ & 0.5 & $2-3$ & $0.25 \mathrm{LT}$ \\
\hline 5 & 0.8 & $2-3$ & $0.5 \mathrm{LT}$ & \begin{tabular}{|l}
0.7 \\
\end{tabular} & $2-3$ & $0.5 \mathrm{LT}$ & 0.6 & $2-3$ & $0.5 \mathrm{LT}$ & 0.5 & $2-3$ & $0.5 \mathrm{LT}$ \\
\hline 5 & 0.8 & $2-3$ & $0.75 \mathrm{LT}$ & \begin{tabular}{|l}
0.7 \\
\end{tabular} & $2-3$ & $0.75 \mathrm{LT}$ & 0.6 & $2-3$ & $0.75 \mathrm{LT}$ & 0.5 & $2-3$ & $0.75 \mathrm{LT}$ \\
\hline 5 & 0.8 & $2-3$ & $1 \mathrm{LT}$ & 0.7 & $2-3$ & 1 1LT & 0.6 & $2-3$ & $1 \mathrm{LT}$ & 0.5 & $2-3$ & $1 \mathrm{LT}$ \\
\hline 5 & 0.8 & $2-3$ & $1.5 \mathrm{LT}$ & 0.7 & $2-3$ & $1.5 \mathrm{LT}$ & 0.6 & $2-3$ & $1.5 \mathrm{LT}$ & 0.5 & $2-3$ & $1.5 \mathrm{LT}$ \\
\hline 5 & 0.8 & $2-3$ & $2.0 \mathrm{LT}$ & \begin{tabular}{|l|l}
0.7 \\
\end{tabular} & $2-3$ & $2.0 \mathrm{LT}$ & 0.6 & $2-3$ & $2.0 \mathrm{LT}$ & 0.5 & $2-3$ & $2.0 \mathrm{LT}$ \\
\hline 5 & 0.8 & $5-0$ & OLT & 0.7 & $5-0$ & OLT & 0.6 & $5-0$ & $0 \mathrm{LT}$ & 0.5 & $5-0$ & $0 \mathrm{LT}$ \\
\hline 5 & 0.8 & $5-0$ & $0.25 \mathrm{LT}$ & 0.7 & $5-0$ & $0.25 \mathrm{LT}$ & 0.6 & $5-0$ & $0.25 \mathrm{LT}$ & 0.5 & $5-0$ & $0.25 \mathrm{LT}$ \\
\hline 5 & 0.8 & $5-0$ & $0.5 \mathrm{LT}$ & \begin{tabular}{|l|l|}
0.7 \\
\end{tabular} & $5-0$ & $0.5 \mathrm{LT}$ & 0.6 & $5-0$ & $0.5 \mathrm{LT}$ & 0.5 & $5-0$ & $0.5 \mathrm{LT}$ \\
\hline 5 & 0.8 & $5-0$ & $0.75 \mathrm{LT}$ & 0.7 & $5-0$ & $0.75 \mathrm{LT}$ & 0.6 & 5-0 & $0.75 \mathrm{LT}$ & 0.5 & $5-0$ & $0.75 \mathrm{LT}$ \\
\hline 5 & 0.8 & $5-0$ & $1 \mathrm{LT}$ & 0.7 & $5-0$ & $1 \mathrm{LT}$ & 0.6 & $5-0$ & $1 \mathrm{LT}$ & 0.5 & $5-0$ & $1 \mathrm{LT}$ \\
\hline 5 & 0.8 & $5-0$ & $1.5 \mathrm{LT}$ & 0.7 & $5-0$ & $1.5 \mathrm{LT}$ & 0.6 & $5-0$ & $1.5 \mathrm{LT}$ & 0.5 & $5-0$ & $1.5 \mathrm{LT}$ \\
\hline 5 & 0.8 & $5-0$ & $2.0 \mathrm{LT}$ & 0.7 & $5-0$ & $2.0 \mathrm{LT}$ & 0.6 & $5-0$ & $2.0 \mathrm{LT}$ & 0.5 & $5-0$ & $2.0 \mathrm{LT}$ \\
\hline
\end{tabular}

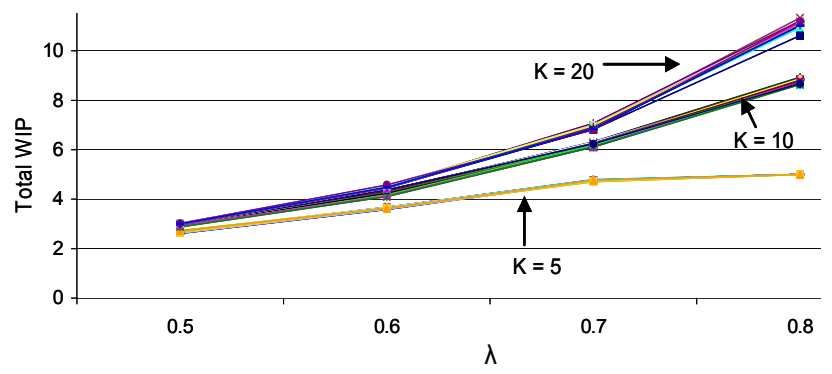

Figure 5: Comparison of WIP vs. $\lambda$ for All the Cases of K, Allocation of K, and Variations of $\tau$.

Figure 6(b) plots the variation of average finished goods inventory, denoted by FG with system load for the same cases. In the legend for the figures, the cases are denoted by the label $10-\tau-3$, implying that $\mathrm{K}=10$ and the initial finished goods inventory is 0 (allocation $\mathrm{K}-0$ ) in all cases.

The demand information lead time, $\tau$ ranges from 0LT (case 10-1-3), 0.5 LT (case 10-3-3), to 1 LT (case 10-5-3). From the plots we make several observations. First, we note that the number of backorders increases significantly with system load. As expected, as the number of backorders increase, the average amount of finished goods inventory reduces (less than one unit in this case). Second, we observe that at high system loads, integrating ADI with pull systems could significantly decrease backorders (thus improving customer service levels). Third, we note that this improved service level is obtained without significant increase in finished goods inventories. This coupled with the observation in Figure 5, that the total average WIP is fairly insensitive to ADI, leads us to conclude that, integration of ADI with pull systems can help significantly improve customer service levels without significant increase in inventory holding costs. For instance, we note that for the case where $\lambda=0.8$, investment in ADI allows us to reduce the average amount of backorders by about $40 \%$, from over 18 units (when $\tau=0 \mathrm{LT}$ ) to a little over 10 units (when $\tau=1 \mathrm{LT}$ ). However, using ADI to potentially build ahead only resulted in an increase in finished goods inventory by roughly 1 unit on the average.

The next figure explores the effect of ADI on average backorders for different initial allocations of finished goods inventories. Figure 7 plots the average backorders against system load $(\lambda)$ for three different cases with $\mathrm{K}=5$ and initial allocations of finished goods inventories corresponding to $0-\mathrm{K}, \mathrm{K} / 2-\mathrm{K} / 2$ and $\mathrm{K}-0$ respectively. In all three cases, we study the impact of ADI on the average number of backorders by considering cases where $\tau$ takes 5 different values (0LT, 0.5LT, 1LT, 1.5LT, 2LT). Figure 7(a) plots the tradeoff when the initial finished goods inventory level is 5 (allocation $0-\mathrm{K}$ ), corresponding to $\mathrm{K}=5$. The specific cases for the different ADI scenarios are denoted by (5-1-1) to (5-7-1) with the case 5-1-1 denoting the case with 5-1-1 and the case 5-7-1 denoting the case with $\tau=2 \mathrm{LT}$. Figure 7(b) plots the tradeoff when the initial finished goods inventory level is 3 (allocation $\mathrm{K} / 2-\mathrm{K} / 2$ ). The 


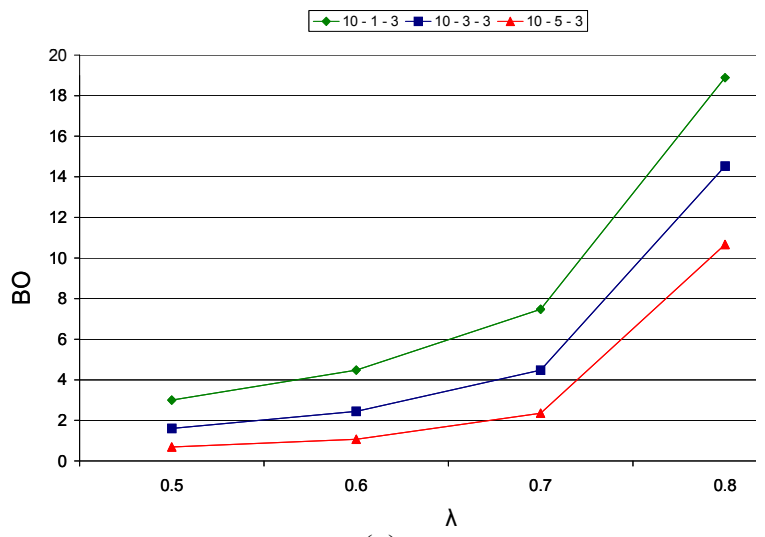

(a)

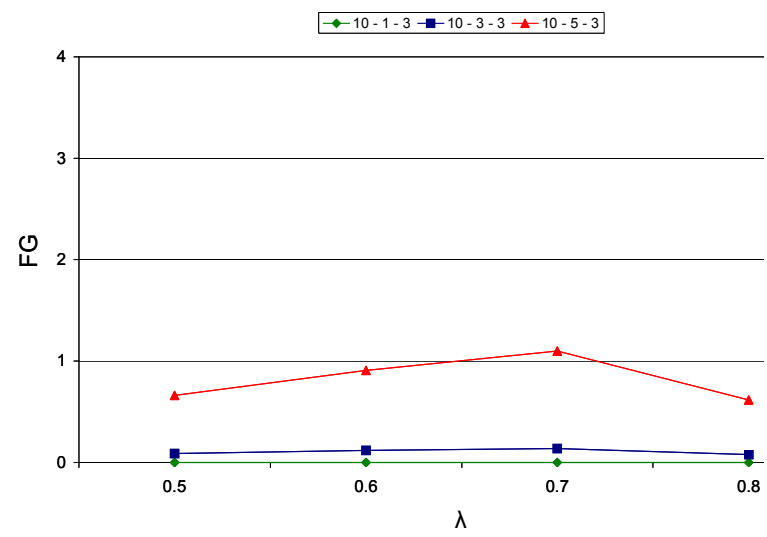

(b)

Figure 6: (a) Comparison of $\mathrm{BO}$ vs. $\lambda$. (b) Comparison of FG vs. $\lambda$. Both Figures Plot the Tradeoff When K=10, Target Finished Goods Inventory is $0(10-\tau-3)$, and $\tau=0 \mathrm{LT}(10-1-3), 0.5 \mathrm{LT}(10-3-3)$, and $1 \mathrm{LT}(10-5-3)$.

specific cases for the different ADI scenarios are denoted by (5-1-2) to case (5-7-2). Finally, Figure 7(c) plots the tradeoff when the initial finished goods inventory level is 0 (allocation K-0) with the specific cases for the different ADI scenarios being denoted by (5-1-3) to case (5-7-3).

The graphs indicate that investment in ADI helps to reduce the average number of backorders. Further, this decrease is fairly independent of the initial allocation of finished goods inventory. Additionally, we observe (as in Figure 6) that as the amount of ADI $(\tau)$ increases, the average number of backorders decrease and that this decrease is more significant at higher loads $(\lambda)$. At lower system loads $(\lambda=0.5)$ we see that the pull systems without ADI are sufficient to ensure that the average number of backorders is less, implying that investments in ADI might not be necessary in these situations. Further, it also appears that the percentage decrease in backorders is a function of the number of kanbans in the system. While investments in ADI yielded a $40 \%$ reduction in backorders for the system settings in Figure 6 with $\mathrm{K}=10$, only $20 \%$ reductions in backorders were obtained via investments in ADI for the system settings in Figure 7 with $\mathrm{K}=5$.

Next, we study this effect of increasing the kanbans cards on backorders in systems with ADI in more detail. Figure 8 plots the average backorders against system load ( $\lambda$ ) for three different cases with $\mathrm{K}=5, \mathrm{~K}=10$ and $\mathrm{K}=20$ respectively. For each of these cases, we consider systems where the initial finished goods inventory is 0 , corresponding to the allocation K-0 of the K kanbans. In all three cases, we study the impact of ADI on the average number of backorders by considering cases where $\tau$ takes 5 different values $(0 \mathrm{LT}, 0.5 \mathrm{LT}, 1 \mathrm{LT}, 1.5 \mathrm{LT}, 2 \mathrm{LT})$. Corresponding to whether, $\mathrm{K}=5,10$ or 20 , the different cases are labeled (5-1-3) to (5-7-3), (10-1-3) to (10-7-3) and (20-1-3) to (20$7-3)$, respectively. From the graphs, we observe that the average number of backorders decreases as $\mathrm{K}$ increases. We see the plots for $\mathrm{K}=5,10$ and 20 in Figure 8 (a), (b) and (c) respectively. It appears that from a system point of view, there are two main options for reducing backorders and improving service levels, namely, investing in ADI, (as suggested by Figure 7) or investing in additional inventory (as suggested by Figure 8). The average number of backorders can be decreased by investing in additional inventory (increasing $\mathrm{K}$ ). However, further reductions in backorders can be achieved by investing in ADI. The graphs also indicate that the beyond a certain point, additional investment in ADI might not yield significant reduction in backorders. For instance, when $\mathrm{K}=10$ and $\mathrm{K}=20$, the reduction in the average number of backorders as $\tau$ increases from $1 \mathrm{LT}$, to $1.5 \mathrm{LT}$ and $2 \mathrm{LT}$, is negligible.

These analyses indicate that the improvements obtained by integrating ADI with pull systems depend upon parameters such as inventory limits, production capacities, and amount of demand information lead time. In order to understand the effect of integrating ADI with pull at an aggregate level, we compare systems in different settings in terms of a total cost (TC) function that include the cost of work in process (WIP), finished goods (FG), and backorders(BO). We write

$$
\mathrm{TC}=\mathrm{h} \times \mathrm{WIP}+\mathrm{f} \times \mathrm{FG}+\mathrm{b} \times \mathrm{BO}
$$

where $\mathrm{h}$ is the unit holding cost, $\mathrm{f}$ is the unit holding cost of finished goods, and $\mathrm{b}$ is the penalty cost for each backordered unit. For our comparison, we assume $\mathrm{h}=\$ 1 / \mathrm{unit}, \mathrm{f}=$ $\$ 2 /$ unit, and $b=\$ 10 /$ unit, although the performance trends reported below hold for other values in this relative range of values for $h, f$ and $b$. After calculating the total cost, we plot the results for the different levels of $\tau$ for two cases of $\lambda$, namely, $\lambda=0.5$ and $\lambda=0.8$.

Figure 9 presents the plots for such cases when $\mathrm{K}=10$ and $K=20$. For each value of $\lambda$ and $K$, we consider the case where the target level of FG inventory is (i) 0 denoted by the label (K-0) and (ii) $\mathrm{K}$ denoted by the label $(0-\mathrm{K})$. 


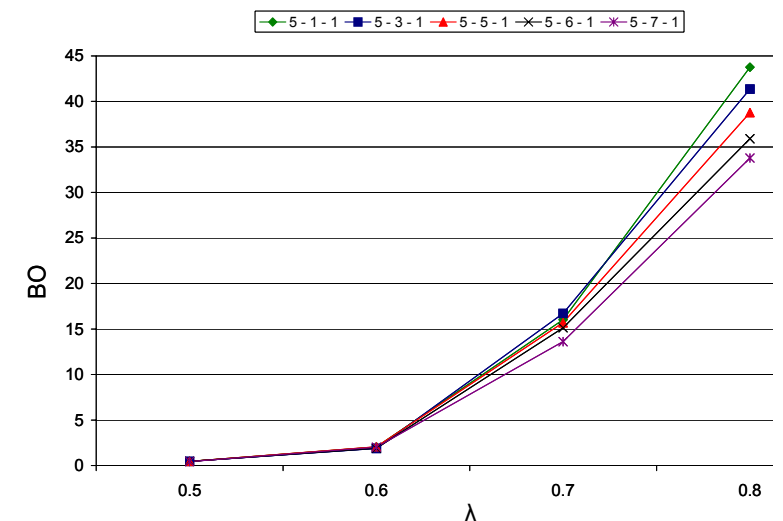

(a)

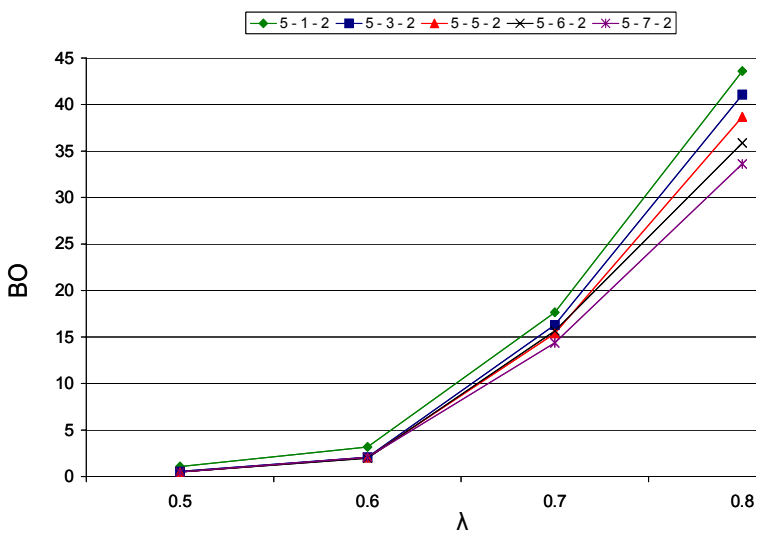

(b)

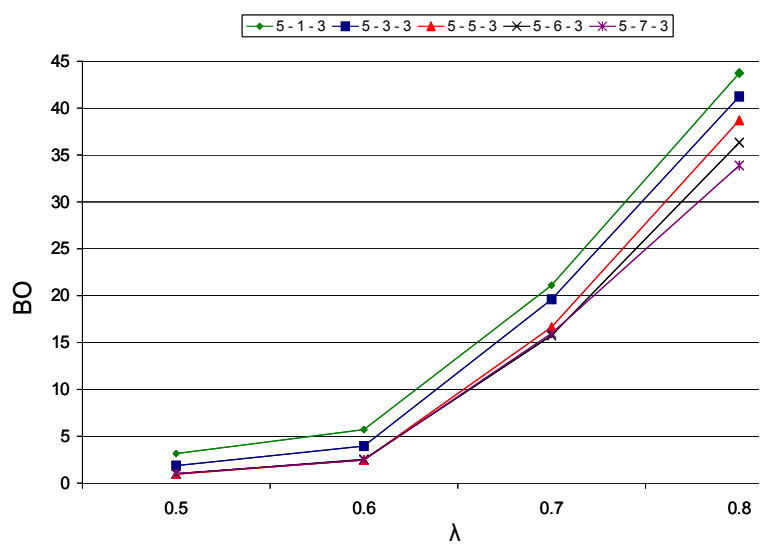

(c)

Figure 7: Comparison of $\mathrm{BO}$ versus $\lambda$ for Different Cases When $\mathrm{K}=5$.

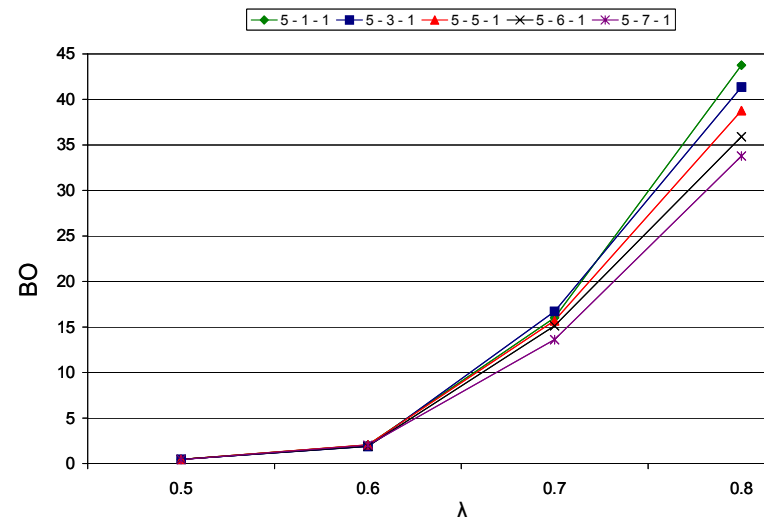

(a)

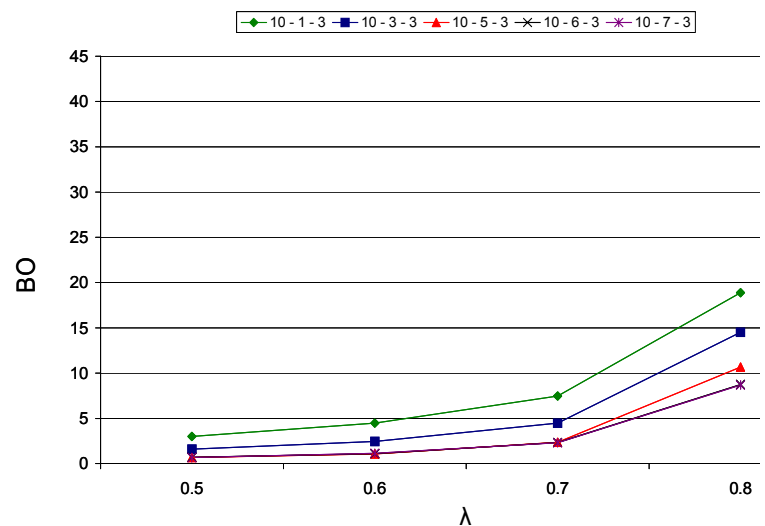

(b)

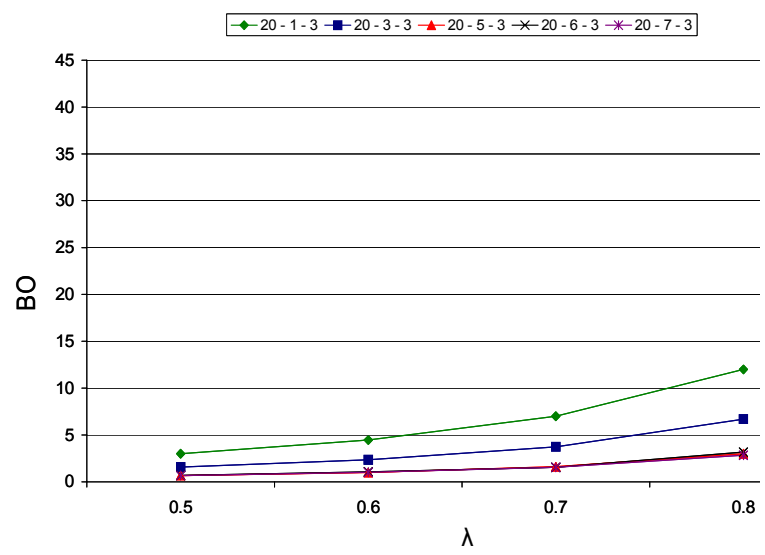

(c)

Figure 8: Comparison of $\mathrm{BO}$ versus $\lambda$ for Different Cases When $\mathrm{K}=5,10$ and 20 


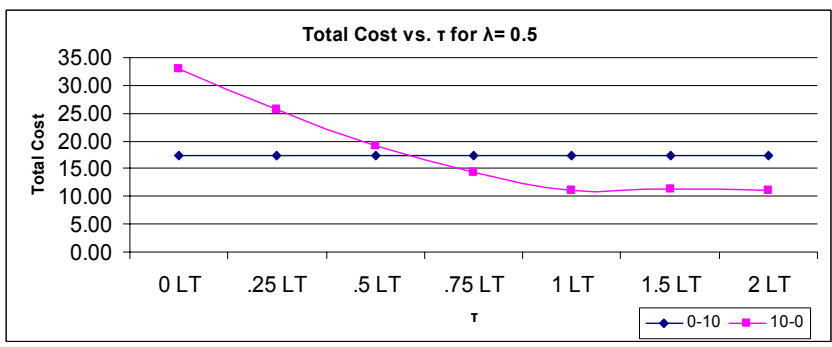

(a)

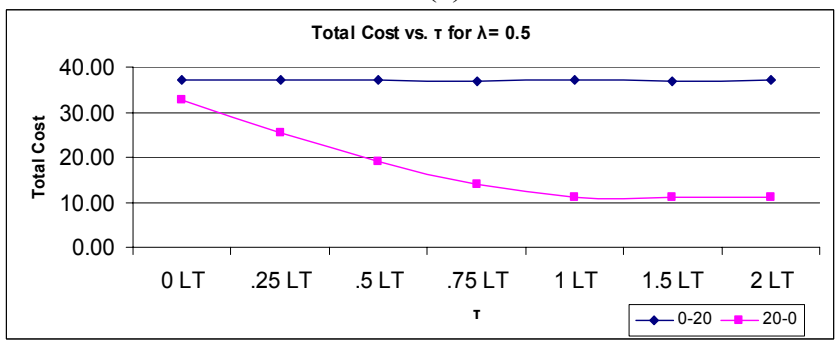

(b)

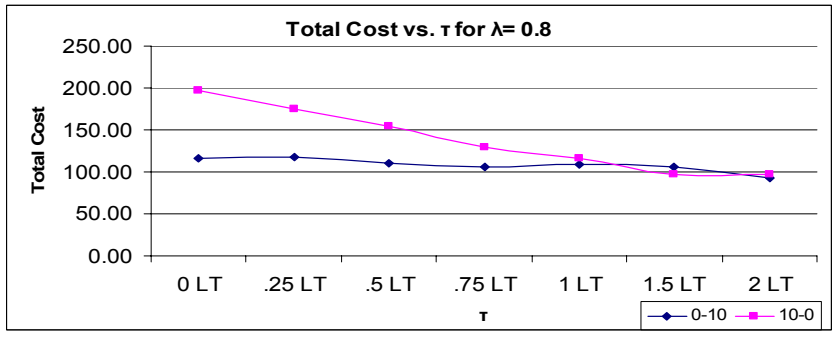

(c)

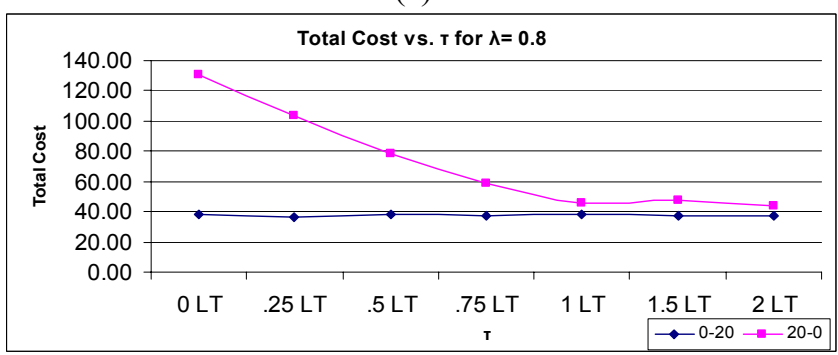

(d)

Figure 9: Total cost for the different levels of $\tau$. a) $\mathrm{K}=10$, $\lambda=0.5$ b) $\mathrm{K}=20, \lambda=0.5$ c) $\mathrm{K}=10, \lambda=0.8$ d) $\mathrm{K}=20, \lambda=0.8$

These plots provide us the following insights. First, we see that when the target finished goods level is $\mathrm{K}$, i.e. case 0 $\mathrm{K}$, investment in ADI does not provide any cost reduction. This is understandable, since in this situation, customers orders are primarily served from existing finished goods stock.

In this case, the system operates in a make to stock mode even in the presence of ADI and the ADI is merely used to replenish consumed inventory. However, we note that there is an inherent disadvantage of operating a system under this policy, since satisfying demands from stock requires prior commitment of expensive production resources and committing systems ability to be responsive to fluctuations in demand. While this strategy might be reasonable and possibly preferable in high loads $(\lambda=0.8)$, this strategy can be highly inefficient at low values of system loads $(\lambda=0.5)$ which could correspond to cases where the market demand is fragment among the different products. The limited effect of ADI at higher loads observed in our simulation study has been observed in other studies on base stock policies with ADI (Karaesman, et al. (2002). Second, it reinforces our earlier observation that in many cases, investment in ADI beyond a certain levels might lot yield significant benefits (see graphs for the case where the target finished goods level is zero.

\section{PULL SYSTEMS WITH IMPERFECT ADI}

This section discusses the simulation results related to systems with imperfect ADI. We focus only on the effect of variance in demand information lead times, i.e., we assume that although demand information is obtained on the average $\tau$ time units in advance, there are no guarantees with respect to demand information lead times for a particular order. For individual orders, the demand information lead time for particular orders is distributed randomly about this mean $\tau$. In particular we assume that this demand information lead time distribution is exponential.

\subsection{Results and Insights}

In order to compare perfect versus imperfect ADI we plot the average number of backorders versus $\mathrm{K}$ for the case where the target finished goods inventory level is 0 (case $\mathrm{K}-0$ ). We do this for the cases where $\tau$ is $0.5 \mathrm{LT}$ and $1 \mathrm{LT}$ and $\lambda=0.8$ and $\lambda=0.5$ respectively. We see from Figures 10 and 11 that as expected, the number of backorders decreases with increase in $\mathrm{K}$ and increase in $\tau$. Further, the benefits of ADI decreases with increase in variance of ADI. Our experiments provide insights into whether supply chain managers should invest in efforts that increases the amount of demand information lead time or invest in efforts that reduces the variability in the demand information lead time provided by the customers. It appears that the performance of the system is less sensitive to variance in demand information lead times.

The figures indicate that even if demand information has significant variation about the mean for particular orders, the average number of backorders (and hence service levels) remain reasonable. The graphs also indicate that the magnitude of the demand information lead time is more important than the variability in this lead time. For instance, a system with imperfect ADI where the demand information lead time is exponentially distributed about a mean of $\tau=\mathrm{LT}$ results in fewer backorder than a system with perfect ADI but with demand information lead time being equal to $\tau=0.5 \mathrm{LT}$ for each order. This robustness of 
system performance against variability in demand information lead times can be of significant value in prioritizing efforts targeted at improving the quality of ADI.

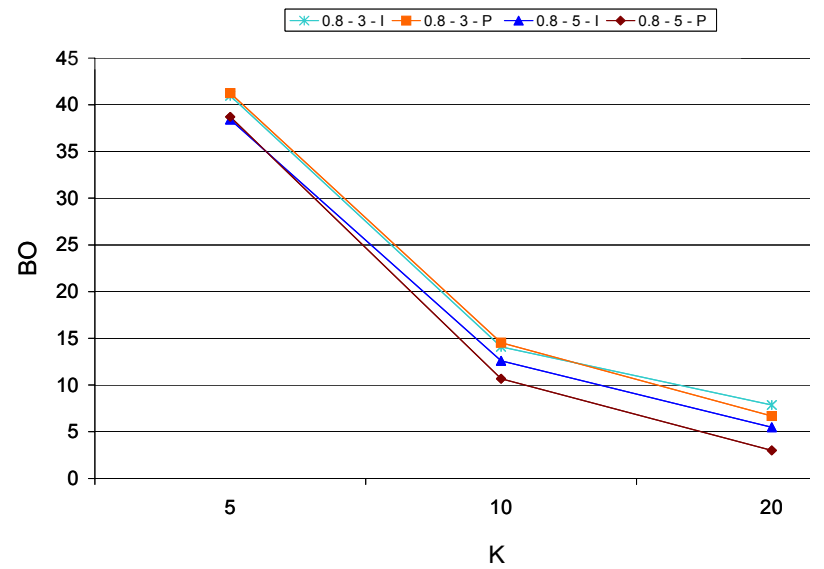

Figure 10: Backorder vs. K Comparing Perfect vs. Imperfect ADI for $\lambda=0.8$, When $\tau=0.5 \mathrm{LT}$ and $1 \mathrm{LT}$.

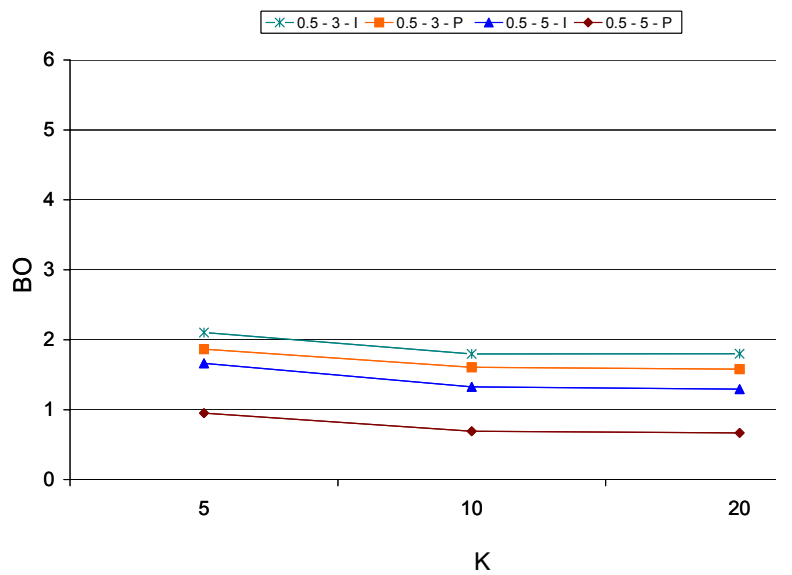

Figure 11: Backorder vs. K Comparing Perfect vs. Imperfect ADI for $\lambda=0.5$, When $\tau=0.5 \mathrm{LT}$ and $1 \mathrm{LT}$.

\section{SUMMARY AND CONCLUSIONS}

In this paper we used simulation experiments to investigate the performance of PICS that integrate ADI with pullkanban type systems. We studied the impact of several design parameters such as kanban card limits, target finished goods inventory levels, and the amount of demand information available on various performance measures. Our study showed that in many situations, integrating ADI with pull systems provides opportunities for efficiencies that might be significantly greater than that available using pull systems alone. Further more, we also showed that the performance of systems operating under such PICS could be fairly robust to the quality of ADI being shared, particularly with respect to the variance in the demand information lead times. Our ongoing research involves investigating the performance of pull systems with ADI in the presence of other imperfections in quality of information and also developing analytical queuing models that will provide more insights into system performance.

\section{REFERENCES}

Buzacott, J.A. and Shanthikumar, J.G., 1993, Stochastic Models of Manufacturing Systems, Prentice Hall, Englewood Cliffs, NJ.

Gallego, G., and Ozer, O., 2001, "Integrating Replenishment Decisions with Advance Order Information," Management Science, Vol. 47, pp. 1344-1360.

Hariharan, R., and Zipkin, P.H., 1995, "Customer-order Information, Lead times and Inventories," Management Science, Vol. 41, pp. 1599-1607.

Hopp, W. and Spearman, M., 1996, Factory Physics: Foundations of Factory Management, Irwin/McGraw Hill, Chicago, IL.

Karaesmen, F., Buzacott, J.A and Dallery, Y., 2002, “Integrating Advance Order Information in Production Control," IIE Transactions, Vol. 34, pp. 649-662.

Krishnamurthy, A., R. Suri and M. Vernon, 2004, "Reexamining the Performance of MRP and Kanban Material Control Strategies for Multi-product Flexible Manufacturing Systems, International Journal of Flexible Manufacturing Systems, Vol. 16, pp. 123-150.

Liberopoulous, G. and Tsikis, I., 2003, "Unified Modeling Framework of Multi-stage Production Inventory Control Policies with Lot sizing and Advance Demand Information," in Stochastic Modeling and Optimization of Manufacturing Systems and Supply Chains, J.G. Shanthikumar, D.D. Yao and W.H.M. Zijm (eds.), International Series in Operations Research and Management Science, Kluwer Academic Publishers.

Zipkin, P.H., 2000, Foundations of Inventory Management, McGrawHill, Boston, MA.

\section{AUTHOR BIOGRAPHIES}

ANANTH KRISHNAMURTHY is an Assistant Professor in the Department of Decision Sciences and Engineering Systems at Rensselaer Polytechnic Institute (RPI). His research interests include: Manufacturing Systems and Supply Chain Management, Queuing Networks and Simulation Optimization. His e-mail is krisha@rpi.edu and his Web address is http: / / www.rpi . edu/ krisha/ .

DAVID CLAUDIO is a PhD student at the Department of Decision Sciences and Engineering Systems at Rensselaer Polytechnic Institute (RPI). His interests include Manufacturing Systems and Supply Chain Management: Lean Manufacturing, Production and Inventory Control, Impact of Information Sharing in Supply Chains His e-mail address is clauddarpi.edu and his Web address is www . freewebs. com/davidclaudioresume/. 